\title{
Dual-Threshold Design of Sub-threshold Circuits
}

\author{
Jia Yao and Vishwani D. Agrawal \\ Department of ECE, Auburn University, Auburn, Alabama 36849 \\ jzy0001@auburn.edu, vagrawal@eng.auburn.edu
}

\begin{abstract}
Dual threshold voltage $\left(V_{t h}\right)$ design is a common method for reducing leakage power in above-threshold circuits. This research shows that it is also effective in reducing energy per cycle of sub-threshold circuits. We first study the single- $V_{t h}$ design theoretically and by simulations, and find that the energy per cycle is independent of threshold voltage. However, in a dual- $V_{t h}$ design, the energy per cycle depends on both threshold voltage and supply voltage. We propose a framework to further reduce energy per cycle below what is possible with a single $V_{t h}$. Given a nominal value for $V_{t h}$, we determine an optimal supply voltage $V_{d d}$ and an optimal higher $V_{t h}$. Application to a 32-bit ripple carry adder shows energy saving of $29 \%$ over the single- $V_{t h}$ lowest energy.
\end{abstract}

\section{INTRODUCTION}

Sub-threshold operation is often referred to as weak inversion operation, where sub-threshold current $I_{s u b}$ is the main source of current. It can be summarized as follows [1]:

$$
I_{s u b}=I_{o} \exp \left(\frac{V_{g s}-V_{t h}}{n V_{t}}\right)\left[1-\exp \left(\frac{-V_{d s}}{V_{t}}\right)\right]
$$

where

$$
I_{o}=\mu C_{o x} \frac{W}{L}(n-1) V_{t}^{2}
$$

$\mu$ is effective mobility, $C_{o x}$ is oxide capacitance, $W$ is transistor width, $L$ is transistor length, $V_{t}$ is thermal voltage, $V_{g s}$ is gate-source voltage, $V_{d s}$ is drain-source voltage, $V_{t h}$ is threshold voltage and $n$ is sub-threshold slope.

Sub-threshold circuits are expected to receive increasing attention in the coming years since the minimum energy CMOS operation occurs in the sub-threshold region [2]. In other words, the optimal supply voltage $\left(V_{d d o p t}\right)$ is typically below $V_{t h}$ when minimum energy is achieved. As the supply voltage scales down into the sub-threshold region, due to the exponential relation between $V_{g s}$ and $I_{s u b}$, circuit delay increases exponentially which causes significant increase in the fraction of leakage energy. On the other hand, dynamic energy decreases relatively slower, i.e., quadratically as supply voltage scales down. Minimum energy point is reached when dynamic energy equals leakage energy.

\section{Single- $V_{t h}$ Sub-Threshold $V_{d d}$ Design}

We establish that energy is independent of $V_{t h}$ for a single$V_{t h}$ and sub-threshold $V_{d d}$ design. However, it is feasible to make the circuit faster without increasing the energy per cycle (EPC) by decreasing $V_{t h}$. An analytical expression for EPC can be written as,

$$
\begin{aligned}
E & =E_{d y n}+E_{\text {leak }}=\frac{1}{2} C_{\text {eff }} V_{d d}^{2}+I_{\text {leak }} V_{d d} T \\
& =\frac{1}{2} C_{e f f} V_{d d}^{2}+I_{o} \exp \left(\frac{-V_{t h}}{n V_{t}}\right) V_{d d} \frac{l C_{g} V_{d d}}{I_{o} \exp \left(\frac{\frac{V_{d d}}{2}-V_{t h}}{n V_{t}}\right)}
\end{aligned}
$$

TABLE I. PTM 32NM [3] $V_{t h}$ CALCUlated By HSPiCE [4].

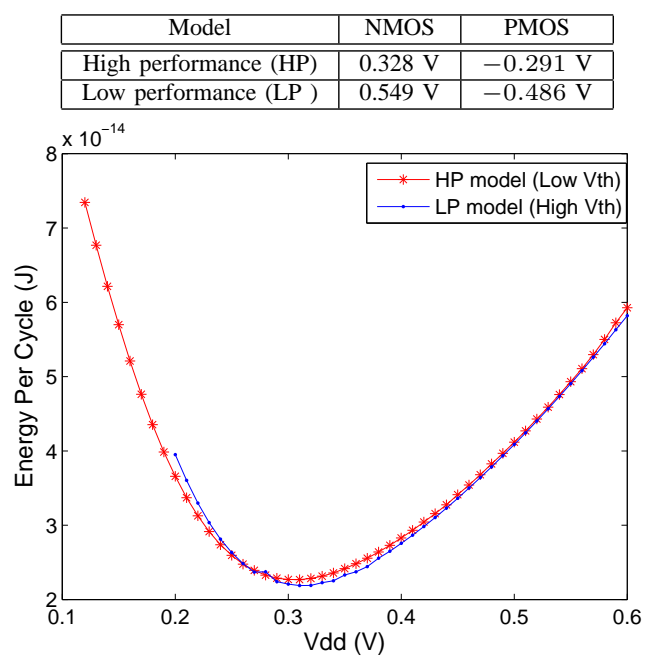

Fig. 1. Random-vector simulation by HSPICE [4] for energy per cycle (EPC) for 32-bit RCA single $V_{t h}$ designs in PTM 32nm CMOS [3] with $W_{n}=5 L$ and $W_{p}=12 L$.

where $C_{\text {eff }}$ is average switched capacitance per clock cycle in the circuit, $C_{g}$ is gate capacitance of a characteristic inverter, $l$ is the length of the critical path in terms of characteristic inverters and $T$ is the clock period. In addition, if we assume that $V_{d s}>3 V_{t}$, so that $1-\exp \left(\frac{-V_{d s}}{V_{t}}\right) \approx 1$, then we arrive at the following expression for energy,

$$
E=\frac{1}{2} C_{e f f} V_{d d}^{2}+\frac{l C_{g} V_{d d}^{2}}{\exp \left(\frac{V_{d d}}{2 n V_{t}}\right)}
$$

From (4) we see that the $V_{t h}$ factor is canceled out in the energy expression which means $V_{t h}$ has no effect on EPC. We verified this theory by simulating a 32-bit ripple carry adder (RCA) in HSPICE. We used PTM 32nm technology [3] which offers two models, a low performance (LP) model with high $V_{t h}$ and a high performance (HP) model with low $V_{t h}$. Table I lists the $V_{t h}$ values calculated at nominal $V_{d d}=0.9 \mathrm{~V}$ by HSPICE [4]. The EPC for the two single threshold voltage circuits as functions of $V_{d d}$ computed by HSPICE [4] (simulating random input vectors) are shown in Figure 1. The red curve is for low $V_{t h}$ and the blue curve is for high $V_{t h}$. We notice that EPC for the two designs remain practically same over the sub-threshold supply voltage range $V_{d d}=0.12 \mathrm{~V}$ to $V_{d d}=0.4 \mathrm{~V}$. As $V_{d d}$ scales down EPC decreases reaching a minimum at the same $V_{d d o p t}$ just above $300 \mathrm{mV}$. When $V_{d d}$ decreases further, EPC increases as leakage energy dominates. Logic operations breakdown earlier, at about $V_{d d}=200 \mathrm{mV}$, for high $V_{t h}$. The low $V_{t h}$ design continues to work at lower $V_{d d}$.

\section{DuAL- $V_{t h}$ Sub-Threshold $V_{d d}$ Design}

In this section, we demonstrate the effectiveness of dual$V_{t h}$ technique as a method to reduce EPC for sub-threshold 


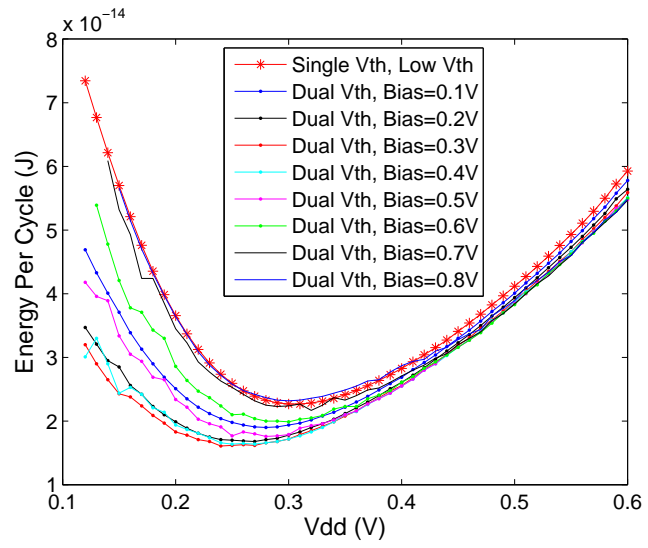

Fig. 2. Random-vector simulation by HSPICE [4] for energy per cycle (EPC) for 32-bit RCA single and dual- $V_{t h}$ designs in PTM 32nm CMOS [3] with $W_{n}=5 L$ and $W_{p}=12 L$.

circuits. Our dual- $V_{t h}$ design keeps circuit speed the same as single- $V_{t h}$ design with low $V_{t h}$, while reducing the leakage power via assigning high $V_{t h}$ to appropriate gates. We use a gate-level slack based algorithm [5], [6] to generate dual- $V_{t h}$ design, consisting of following steps:

1) Assign low $V_{t h}$ to all gates.

2) Run static timing analysis (STA) to calculate slack [5] for each gate and circuit delay $(T)$ for every $V_{d d}$ and high $V_{t h}$ condition.

3) Using gate slacks assign gates to high $V_{t h}$ such that critical path delay does not degrade.

4) Estimate EPC, $V_{\text {ddopt }}$ and an optimal high $V_{t h}$ level which gives the lowest EPC. To estimate EPC, we sum up the energy of all gates, $i=1, \cdots n$. as shown below, where $C_{\text {eff }}$ and $P_{\text {leak }}$ are obtained from HSPICE [4] for basic logic gates under varying $V_{d d}, V_{t h}$ and fan-out conditions.

$$
E=\sum_{i=1}^{n} E_{i}=\alpha C_{e f f, i} V_{d d}^{2}+P_{l e a k, i} T
$$

5) Simulate dual- $V_{t h}$ design [4] and compare EPC, $V_{d d o p t}$ and optimal high $V_{t h}$.

Figure 2 shows how EPC is lowered via optimized dual$V_{t h}$ design. Supply voltage ranges from $0.12 \mathrm{~V}$ to $0.6 \mathrm{~V}$ and we apply reverse body bias voltages to the example circuit in the range between $0.1 \mathrm{~V}$ to $0.8 \mathrm{~V}$. For any given $V_{d d}$, EPC decreases as bias voltage increases until it reaches a lower bound. Then it starts to increase slowly, finally reaching the same value as the single- $V_{t h}$ design. The lowest minimum energy occurs when the bias voltage equals $0.3 \mathrm{~V}$. The minimum EPC in Figure 2 is $1.610 \times 10^{-14} \mathrm{~J}$ at $V_{d d o p t}=0.24 \mathrm{~V}$. The corresponding critical path delay is $T=1.2134 \mu \mathrm{s}$, resulting in a clock frequency of $0.8241 \mathrm{MHz}$.

In comparison, for the two single-threshold designs of Figure 1 the minimum EPC is $2.268 \times 10^{-14} \mathrm{~J}$ at $V_{d d o p t}=0.31 \mathrm{~V}$. For low $V_{t h}$ circuit, $T=250.11 \mathrm{~ns}$ or clock frequency = $3.998 \mathrm{MHz}$, and for high $V_{t h}$ circuit, $T=35.835 \mu$ s or clock frequency $=27.9 \mathrm{kHz}$. Thus, EPC for the dual- $V_{t h}$ circuit is $29.1 \%$ lower than that for either of the single- $V_{t h}$ circuit. The speed of the dual- $V_{t h}$ circuit is between the speeds of the two single- $V_{t h}$ circuits.

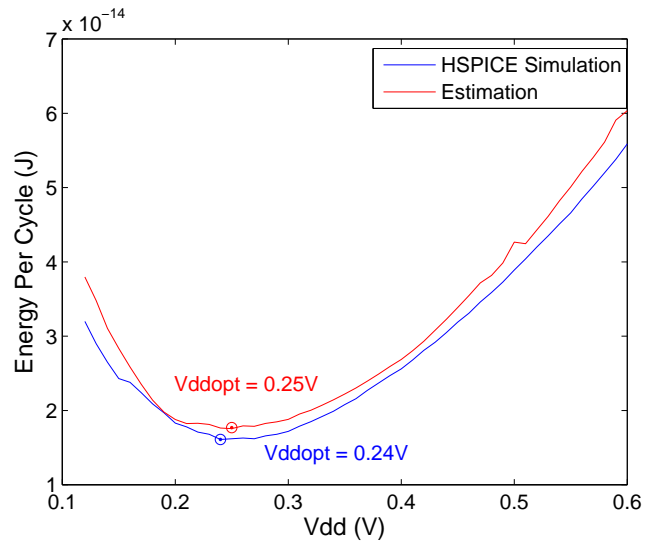

Fig. 3. Random-vector HSPICE [4] simulation results vs. estimation results for energy per cycle (EPC) for 32-bit RCA dual- $V_{t h}$ design with reverse body bias voltage $=0.3 \mathrm{~V}$.

Figure 3 compares estimated EPC of dual- $V_{t h}$ designs with HSPICE [4] simulation. The average error between the estimation and simulation is $6.99 \%$. The error may result from simplifications made in the framework. For example, we assume that fan-out gates are always low $V_{t h}$ gates when calculating output capacitance of the driving gate in HSPICE [4]. That is, when a gate drives high $V_{t h}$ gates, the difference in output capacitance is considered negligible.

Acknowledgment - This research is supported by the Wireless Engineering Research and Education Center at Auburn University.

\section{CONCLUSION}

We use the energy per cycle (EPC) as a measure of efficiency of a CMOS circuit. It is known that minimum EPC for CMOS circuits occurs when $V_{d d}$ has a sub-threshold value. We show that this optimum operation remains unchanged as the threshold voltage $\left(V_{t h}\right)$ of devices is varied in a single threshold circuit. The reason is that an increase of $V_{t h}$ increases the delay and decreases the leakage current in similar proportions. We find a new minimum for EPC of the subthreshold $V_{d d}$ circuit achieved by our dual- $V_{t h}$ design. As an example, EPC of a 32-bit ripple carry adder in 32nm CMOS is lowered by $29 \%$ over its single threshold version.

\section{REFERENCES}

[1] A. Wang, B. H. Calhoun, and A. P. Chandrakasan, Sub-Threshold Design for Ultra Low-Power Systems, Springer, 2006

[2] A. Wang and A. P. Chandrakasan, "A 180mV FFT Processor Using Subthreshold Circuit Techniques," in Proc. IEEE International SolidState Circuits Conf., Feb. 2004, pp. 292-295.

[3] "Latest PTM Models." Arizona State University, http://ptm.asu.edu/ (accessed on Dcember 11, 2011).

[4] "HSPICE Reference Manual: Commands and Control Options, Version D-2010.03-SP1," June 2010. http://www.synopsys.com/Tools/Verification/AMSVerification/CircuitSimulation/HSPICE/Pages/default.aspx (accessed on October 14, 2011).

[5] K. Kim and V. D. Agrawal, "Ultra Low Energy CMOS Logic Using Below-Threshold Dual-Voltage Supply," in Jour. of Low Power Electronics, vol. 7, no. 4, pp. 460-470, December 2011.

[6] M. Allani and V. D. Agrawal, "An Efficient Algorithm for Dual-Voltage Design Without Need for Level Conversion," in Proceedings of IEEE Southeastern Symp. on System Theory, March 2012, pp.51-56. 